INTERVENTIONAL CARDIOLOGY AND SURGERY

\title{
One year cost effectiveness of sirolimus eluting stents compared with bare metal stents in the treatment of single native de novo coronary lesions: an analysis from the RAVEL trial
}

\author{
B A van Hout, P W Serruys, P A Lemos, M J B M van den Brand, G-A van Es, W K Lindeboom, \\ M-C Morice
}

Heart 2005;91:507-512. doi: 10.1136/hrt.2004.034454

\begin{abstract}
Objective: To assess the balance between costs and effects of the sirolimus eluting stent in the treatment of single native de novo coronary lesions in the RAVEL (randomised study with the sirolimus eluting Bx Velocity balloon expandable stent in the treatment of patients with de novo native coronary artery lesions) study.

Design: Multicentre, double blind, randomised trial

Setting: Percutaneous coronary intervention for single de novo coronary lesions

Patients: 238 patients with stable or unstable angina.

Interventions: Randomisation to sirolimus eluting stent or bare stent implantation.

Main outcome measures: Patients were followed up to one year and the treatment effects were expressed as one year survival free of major adverse cardiac events (MACE). Costs were estimated as the product of resource utilisation and Dutch unit costs.

Results: At one year, the absolute difference in MACE-free survival was $23 \%$ in favour of the sirolimus eluting stent group. At the index procedure, sirolimus eluting stent implantation had an estimated additional procedural cost of $€ 1286$. At one year, however, the estimated additional cost difference had decreased to $€ 54$ because of the reduction in the need for repeat revascularisations in the sirolimus group $10.8 \%$ v 23.6\%; $p<0.01)$. After adjustment of actual results for the consequences of angiographic follow up (correction based on data from the BENESTENT (Belgium Netherlands stent) II study), the difference in MACE-free survival was estimated at $11.1 \%$ and the additional one year costs at $€ 166$.

Conclusions: The one year data from RAVEL suggest an attractive balance between costs and effects for sirolimus eluting stents in the treatment of single native de novo coronary lesions. The cost effectiveness of drug eluting stents in more complex lesion subsets remains to be determined.
\end{abstract}

See end of article for authors' affiliations

Correspondence to: Professor Patrick W Serruys, Thoraxcentre, $\mathrm{Bd}-406, \mathrm{Dr}$

Molewaterplein 40,

3015-GD Rotterdam,

Netherlands;

p.w.j.c.serruys@

erasmusmc.nl

Accepted 8 May 2004
$\mathrm{S}$ irolimus eluting stents have been recently proved to greatly reduce coronary restenosis and the need for subsequent revascularisation compared with conventional stenting. ${ }^{1-3}$ Sirolimus eluting stents have been available for routine use since April 2002 in Europe and since April 2003 in the USA. Owing to the clear clinical superiority of these devices over bare metal stents, clinicians would be expected to want to use the new stent as extensively as possible. However, sirolimus stents are more costly than conventional bare stents, which may increase up front procedural costs and has been perceived as an important limitation to more widespread utilisation of these devices in clinical practice. On the other hand, the reduction of repeat intervention procedures during follow up may save costs, which may eventually lower total costs. Therefore, in the current era of cost containment policies, the question arises as to how the additional effects compare with the additional costs.

The present study addressed this question for patients included in the RAVEL (randomised study with the sirolimus eluting Bx Velocity balloon expandable stent in the treatment of patients with de novo native coronary artery lesions) study. It is important to note that the RAVEL study had a protocol mandated angiogram scheduled at 5-7 months of follow up.' It is known from the Belgium Netherlands stent (BENESTENT) II study that this policy may bias both cost and effectiveness estimates of the treatments when compared with estimates for patients without routine angiographic follow up. ${ }^{4}$ Since routine angiographic follow up is not standard practice in the "daily life" of most centres, the question needs to be raised whether the results would differ without angiographic follow up. This issue was addressed in this report by an analysis that combined estimates of costs and effects from the RAVEL study (comparing drug eluting with bare stents) with estimates of the effect of angiographic follow up from the BENESTENT II study (comparing results with and without scheduled angiographic follow up).

\section{MATERIAL AND METHODS}

The study protocol and main findings of the RAVEL study have been reported in detail elsewhere. 'In brief, RAVEL was a randomised, double blind study of 238 patients with a diagnosis of stable or unstable angina scheduled for treatment of a single de novo target lesion in a native coronary vessel. Patients were treated with either a bare metal Bx Velocity stent (Cordis Corp, Miami Lakes, Florida, USA) or a

Abbreviations: ARTS, arterial revascularisation therapies study; BENESTENT, Belgium Netherlands stent; DEBATE, Doppler endpoints balloon angioplasty trial Europe; MACE, major adverse cardiac events; RAVEL, randomised study with the sirolimus eluting Bx Velocity balloon expandable stent in the treatment of patients with de novo native coronary artery lesions 
similar sirolimus eluting Bx Velocity stent. Both stents were indistinguishable, except under microscopy. At 30 days and six and 12 months, patients returned for evaluation and were specifically questioned to identify the possible interim development of angina, as well as to monitor major adverse cardiac events (MACE) including additional revascularisation of the index target lesion. Diagnostic angiography was performed at 180 (range 150-210) days or preceding a reintervention. The decision to perform a reintervention was left to the investigator's discretion, who was asked to register whether it was based on clinical symptoms or guided by angiographic results.

\section{Cost effectiveness}

Effectiveness was assessed by using the composite of 12 month MACE, which were all-cause death, non-fatal myocardial infarction, and target lesion revascularisation (either surgical or percutaneous). With respect to costs, the analysis was limited to the direct medical costs. The balance between costs and effects after 12 months was assessed by computing the incremental cost effectiveness ratio (the average one year costs per patient treated with drug eluting stents minus the average one year costs with bare stent implantation divided by the percentage change in MACE-free survivors after one year).

For cost effectiveness, two scenarios were investigated. The first scenario reflects the actual protocol driven resource use and effectiveness observed in the RAVEL study (which included a six month angiogram). For the estimation of the costs and effects of this scenario, we used methods similar to those used in the assessments of costs and effects in the BENESTENT II study, ${ }^{4}$ the DEBATE (Doppler endpoints balloon angioplasty trial Europe) II study, ${ }^{5}$ and ARTS (arterial revascularisation therapies study). ${ }^{6}$ Resource utilisation data were collected from the case record form for the initial procedure (number of balloons, type and number of stents, type and number of catheters, etc), hospital admissions (coronary care unit, intensive care unit, conventional ward), and major treatment and diagnostic procedures after the initial procedure. Unit costs were estimated, before the analysis of the data, on the basis of detailed information from the Erasmus Medical Centre, Rotterdam, the Netherlands, following an approach similar to that reported previously. ${ }^{7}$ Costs per patient were calculated as the product of each patient's resource utilisation and the corresponding unit cost. Information about the prices of sirolimus eluting and bare stents was obtained from the manufacturing company. In both arms, the medication costs include those of eight weeks of antiplatelet treatment.

The second scenario excluded follow up angiography as a standard procedure. The rationale for this approach was to exclude the effect of the so-called oculostenotic reflex, which may increase the incidence of repeat intervention in patients undergoing protocol mandated angiographic re-evaluation. ${ }^{4} 8$ It is noteworthy that the information collected at the time of the repeat revascularisations on whether the new intervention was driven by angina was indicative of the effect of angiographic follow up. However, these records were not informative about the impact of follow up angiograms on the number of angiographies and repeat procedures that would have taken place before and after 5-7 months. It is expected that some of the procedures performed during the follow up visit would normally have been carried out during the months either before or after the protocol driven angiogram. With respect to the angiograms and interventions that would have been performed before the prespecified time point, it is expected that these were postponed knowing that angiograms were already scheduled at the 5-7 month follow up. One would therefore expect a lower revascularisation rate before a prespecified angiographic follow up than would have been the case without such a specification. Conversely, if an angiogram is prespecified, there may be less need for later angiography and for late repeat procedures than without angiographic follow up.

In the BENESTENT II study, ${ }^{4}$ which compared balloon angioplasty with stenting, a one to one subrandomisation was carried out assigning patients either to planned angiographic follow up (as in the RAVEL trial) or to clinical follow up alone (which is preferred for the purpose of a cost effectiveness analysis). Among patients with protocol mandated angiographic follow up, the rate of repeat revascularisation was $18.27 \%$ (stenting) versus $22.28 \%$ (balloon). Without angiographic follow up, the percentage of repeat revascularisations was $7.77 \%$ versus $16.26 \%$, respectively. With angiographic follow up, the percentage of patients with unscheduled angiograms was $8.17 \%$ versus $12.86 \%$ (with and without stenting). Without angiographic follow up the percentage of unscheduled angiograms was $14.08 \%$ versus $20.20 \%$. Thus, on average, the inclusion of angiographic follow up increased the number of repeat revascularisations by a factor 1.6 and decreased the number of unscheduled angiograms by a factor of 0.6. The data from the BENESTENT II trial, therefore, offer a source for estimating the effect of angiographic follow up on both the occurrence of angiograms and repeat interventions. Moreover, that trial also offers information about the timing of these procedures.

To estimate the effects of angiographic follow up on treatment, the time after the initial procedure was divided into three periods: firstly, from the index procedure to month 5; secondly, from month 5 to month 7; and thirdly, from month 7 to month 12. For each period, the rates of nonscheduled angiographies and of repeat revascularisation were estimated. Differences were estimated in terms of relative risk ratios; those significantly different from 1.0 were included in the analysis. Subsequently, the numbers of patients free of repeat revascularisations were estimated by multiplying the number of repeat revascularisations observed in RAVEL with the relative risk ratios derived from BENESTENT for all three periods. Finally, average MACEfree survival was estimated by multiplying the revascularisation rate times the relative risk scores of patients who only had repeat revascularisations. Patients who died or had a myocardial infarction continued to be counted as having had an event.

A similar procedure was followed with respect to costs. For this analysis, estimates were needed of the costs associated with a repeat procedure and the costs of an angiogram, not only of the procedure itself but also of the additional costs associated with these procedures. These costs were estimated by using the data from the RAVEL study and applying a linear regression analysis with costs as the dependent variable and the various events as independent variables. It was noted that the resulting cost estimates were not just for the cost of the procedure but that they had to be interpreted as the additional costs associated with the treatment of a patient who undergoes such procedures. Total costs were corrected on the basis of the increase in the expected numbers of non-scheduled angiograms and the expected decrease in the number of repeat revascularisations.

\section{Statistical analysis}

All analyses were based on the intention to treat principle. In estimating the risk ratios from BENESTENT II, whether these relative risks differed between the randomised treatments (stent or balloon) was assessed by testing for differences in the log transformed risk ratios. Where the hypothesis of a similar effect could not be rejected (95\% significance), data from both procedures were pooled to estimate the relative 
Table 1 Costs associated with observed resource use

\begin{tabular}{|c|c|c|c|c|c|c|}
\hline & \multicolumn{2}{|c|}{ Resource use per patient } & \multirow[b]{2}{*}{ Unit cost $(€)$} & \multicolumn{3}{|l|}{ Cost $(€)$} \\
\hline & $\begin{array}{l}\text { Sirolimus stent } \\
(\mathrm{n}=120)\end{array}$ & $\begin{array}{l}\text { Bare stent } \\
(n=118)\end{array}$ & & $\begin{array}{l}\text { Sirolimus stent } \\
(\mathrm{n}=120)\end{array}$ & $\begin{array}{l}\text { Bare stent } \\
(n=118)\end{array}$ & Difference \\
\hline \multicolumn{7}{|l|}{ Index procedure } \\
\hline Procedure time (min) & 70.8 & 70.6 & 18 & 1288 & 1285 & 3 \\
\hline Study stent & 1.03 & 1.02 & $2000 / 672$ & 2050 & 683 & 1367 \\
\hline Other stent type & 0.02 & 0.03 & 712 & 12 & 24 & -12 \\
\hline Guiding catheter & 1.10 & 1.07 & 98 & 107 & 104 & 3 \\
\hline Guidewire & 1.08 & 1.04 & 115 & 124 & 120 & 4 \\
\hline Balloon & 1.32 & 1.37 & 491 & 646 & 674 & -28 \\
\hline Doppler & 0.03 & 0.06 & 523 & 13 & 31 & -18 \\
\hline IVUS catheter & 0.23 & 0.29 & 614 & 143 & 177 & -34 \\
\hline Contrast medium & 192.08 & 200.18 & 1 & 98 & 102 & -4 \\
\hline Procedure related medication & & & & 67 & 63 & -4 \\
\hline CCU stay (days) & 0.30 & 0.26 & 963 & 289 & 252 & 37 \\
\hline ICU stay (days) & 0.29 & 0.29 & 1058 & 307 & 305 & 2 \\
\hline Non-CCU/ICU stay (days) & 2.12 & 2.24 & 343 & 727 & 768 & $-4 \overline{1}$ \\
\hline Total procedure costs & & & & 5872 & 4588 & 1284 \\
\hline \multicolumn{7}{|l|}{ Follow up } \\
\hline \multicolumn{7}{|l|}{ Repeat revascularisation } \\
\hline Repeat PTCA (target and non-target) & 0.03 & 0.31 & & 107 & 908 & -800 \\
\hline CABG (target and non-target) & 0.01 & 0.01 & 7448 & 62 & 126 & -64 \\
\hline \multicolumn{7}{|l|}{ Coronary angiography } \\
\hline Non-scheduled angiographies & 0.08 & 0.10 & 2160 & 180 & 220 & -40 \\
\hline Protocol angiographies & 0.90 & 0.74 & 2160 & 1944 & 1592 & 351 \\
\hline Transfusion & 0.01 & 0.01 & 60 & 1 & 1 & 0 \\
\hline Vascular surgery & 0.01 & 0.02 & 4341 & 36 & 74 & -37 \\
\hline Emergency room visit & 0.15 & 0.12 & 44 & 7 & 5 & 1 \\
\hline Observation unit $<24 \mathrm{~h}$ after admission & 0.02 & 0.20 & 193 & 3 & 39 & -36 \\
\hline Outpatient rehabilitation & 0.08 & 0.18 & 23 & 2 & 4 & -2 \\
\hline CCU stay (days) & 0.17 & 0.64 & 963 & 161 & 612 & -452 \\
\hline ICU stay (days) & 0.14 & 0.11 & 1058 & 151 & 117 & 35 \\
\hline Non-CCU/ICU stay (days) & 2.15 & 2.50 & 343 & 737 & 858 & -121 \\
\hline Rehabilitation & 0.24 & 0.37 & 343 & 83 & 128 & -45 \\
\hline Total follow up costs & & & & 3473 & 4683 & -1210 \\
\hline Total direct medical cost (excluding medication) & & & & 9345 & 9271 & 74 \\
\hline Medication & & & & 624 & 644 & -20 \\
\hline Total direct medical cost (including medication) & & & & 9969 & 9915 & 54 \\
\hline
\end{tabular}

risks. A correction for the number of angiograms and repeat procedures was made when the risk ratios differed $(95 \%$ significance) from 1.

With respect to the scenario assuming no angiographic follow up, the uncertainties surrounding the estimate were addressed by a combination of bootstrapping and multivariate sensitivity analysis. In bootstrapping, a number, say 1000, of new trials are simulated of exactly the same size as the original trial. This is done by drawing patients at random (with replacement) from the original trial. Each bootstrap leads to a new estimate of average costs and average effects. The resulting 1000 estimates can be summarised in terms of a distribution. Truncating the upper and lower $2.5 \%$ provides the $95 \%$ confidence intervals. This is a very convenient method when the distribution cannot be obtained in a classic way. ${ }^{9}$

\section{RESULTS}

In total, 120 patients were randomly assigned to sirolimus eluting stent implantation and 118 patients to bare metal

Table 2 Non-scheduled angiograms and repeat revascularisation in the BENESTENT ॥ trial $^{4}$

\begin{tabular}{|c|c|c|c|}
\hline & $\begin{array}{l}\text { Without angiographic } \\
\text { follow up (angioplasty } \\
\text { with and without stents) }\end{array}$ & $\begin{array}{l}\text { With angiographic follow } \\
\text { up (angioplasty with and } \\
\text { without stents) }\end{array}$ & Relative risk $(95 \% \mathrm{CI})$ \\
\hline \multicolumn{4}{|l|}{ Months 1-5 } \\
\hline Angiograms & $8.6 \%$ & $7.2 \%$ & $1.192(0.747$ to 1.904$)$ \\
\hline Repeat revascularisations & $6.1 \%$ & $8.9 \%$ & $0.691(0.424$ to 1.126$)$ \\
\hline \multicolumn{4}{|l|}{ Months 6-7 } \\
\hline Angiograms & $3.4 \%$ & $1.0 \%$ & $3.577(1.187 \text { to } 10.776)^{*}$ \\
\hline Repeat revascularisations & $3.4 \%$ & $4.5 \%$ & $0.387(0.212 \text { to } 0.704)^{*}$ \\
\hline \multicolumn{4}{|l|}{ Month 8-12 } \\
\hline Angiograms & $5.1 \%$ & $2.4 \%$ & $2.146(1.023 \text { to } 4.501)^{*}$ \\
\hline Repeat revascularisations & $2.4 \%$ & $2.6 \%$ & $0.929(0.399$ to 2.164$)$ \\
\hline \multicolumn{4}{|c|}{$\begin{array}{l}\text { Differences of log transformed risk ratios were not significant based on the } 95 \% \text { confidence interval (CI) } \\
\text { surrounding these differences. } \\
\text { BENESTENT, Belgium Netherlands stent. } \\
\text { *Significant risk ratio used in RAVEL (randomised study with the sirolimus eluting Bx Velocity balloon expandable } \\
\text { stent in the treatment of patients with de novo native coronary artery lesions) cost effectiveness model. }\end{array}$} \\
\hline
\end{tabular}




\begin{tabular}{|c|c|c|}
\hline & Cost estimate $(€)$ & Standard error \\
\hline Initial costs & 5097 & 253 \\
\hline Additional costs of drug eluting stent & 1360 & 311 \\
\hline Death* & 1194 & 1197 \\
\hline Myocardial infarction & 3693 & 788 \\
\hline Coronary bypass surgery & 12147 & 1316 \\
\hline First repeat percutaneous intervention & 4374 & 455 \\
\hline Additional repeat percutaneous intervention & 6665 & 705 \\
\hline Non-scheduled angiography & 3561 & 538 \\
\hline Other serious adverse events & 4411 & 246 \\
\hline
\end{tabular}

stents. With the exception of a higher percentage of men in the control arm, the two groups had similar baseline and procedural characteristics. At one year, the sirolimus and bare stent group had similar mortality (both $1.7 \%$ ) and myocardial infarction rates $(3.3 \% \vee 4.2 \%$, respectively). The one year incidence of MACE was significantly reduced in the sirolimus group compared with the controls $(5.8 \% v 28.8 \%$, $p<0.01$ by log rank test), mainly due to a major decrease in the need for repeat revascularisation in the sirolimus group $(0 \% \vee 22.9 \%$, $\mathrm{p}<0.01)$. Table 1 presents the estimates of costs after one year. It appears that the additional costs of the initial procedure were almost completely recouped by the decrease in the costs of follow up. Based on the observed event rates and overall costs in RAVEL, without correcting for the impact of protocol mandated follow up angiograms, at the end of the first year the total costs were estimated to be only $€ 54$ per patient higher in the sirolimus eluting stent group than in the bare stent group. Costs per MACE-free survivor were estimated at $€ 234$ with an upper $95 \%$ limit of $€ 5679$.

When considering the results in table 1 it may be noted that the costs of the scheduled angiograms are higher in the sirolimus group than in the bare stent group (which is related to the higher number of patients with repeat procedures in the bare stent group). This may suggest that without such follow up, the costs of the initial procedure would have been completely recouped. However, this suggestion would neglect the effect of the oculostenotic reflex, since the difference in repeat percutaneous revascularisation procedures between the two groups suggests that such a reflex may have had an effect on the costs and the outcomes.

Table 2 summarises the frequency of non-scheduled angiograms and repeat revascularisation procedures in patients with and without protocol mandated follow up angiography in the BENESTENT II trial. ${ }^{4}$ The need for nonscheduled angiography in each group is shown according to the time of its occurrence before, during, or after the period in which follow up angiograms were scheduled. There were no significant differences in the relative risk ratios for nonscheduled angiography in the stent and balloon arms in BENESTENT II. As table 2 shows (pooled data of the balloon and stent groups), protocol mandated angiographic follow up had no significant effect on the risk of unscheduled angiography during the first five months, and no correction factor was applied for this period. However, between five and seven months (period of the scheduled angiography), patients with no protocol mandated angiography had significantly more non-scheduled angiograms (increased by a factor of 3.577) and fewer repeat procedures (reduced by a factor of 0.387 ). Also, after seven months, the group with protocol mandated angiography had more non-scheduled angiograms, which were accordingly corrected by a factor of 2.146. After correction of the RAVEL data according to the expected effects of angiographic follow up, the difference in the number of repeat procedures was then estimated at $11.8 \%$ instead of $23.6 \%$. Moreover, the difference in the number of unscheduled angiograms was estimated at 3.8\% instead of $1.9 \%$.

Table 3 shows the results from the regression analyses relating the costs per patient to the costs of the initial procedure and the occurrence of all MACE, other serious adverse events, and angiograms $\left(r^{2}=0.35 ; F=10.76\right.$; $F$ test $\mathrm{p}<0.001)$. Applying the derived relative risks to the patient specific data leads to the estimates of both costs and effects as presented in table 4 . Without routine angiographic follow up the difference in costs between the sirolimus eluting stent and the bare metal stent at one year was estimated to be $€ 166$. The costs per additional MACE-free survivor are now estimated to be $€ 1495$ with an upper $95 \%$ limit of $€ 61243$. Figure 1 presents the estimates of both cost and effects after a combination of bootstrapping and

Table 4 Costs and effects based on various scenarios

\begin{tabular}{|c|c|c|c|c|c|c|}
\hline & \multicolumn{3}{|c|}{ With angiographic follow up } & \multicolumn{3}{|c|}{ Without angiographic follow up } \\
\hline & $\begin{array}{l}\text { Sirolimus stent } \\
(n=120)\end{array}$ & $\begin{array}{l}\text { Bare stent } \\
(n=118)\end{array}$ & Difference & $\begin{array}{l}\text { Sirolimus stent } \\
(n=120)\end{array}$ & $\begin{array}{l}\text { Bare stent } \\
(n=118)\end{array}$ & Difference \\
\hline \multicolumn{7}{|l|}{ Clinical events } \\
\hline Death & $1.7 \%$ & $1.7 \%$ & 0 & $1.7 \%$ & $1.7 \%$ & 0 \\
\hline Myocardial infarction & $3.3 \%$ & $5.1 \%$ & $-1.8 \%$ & $3.3 \%$ & $5.1 \%$ & -1.8 \\
\hline Tárget lesion revascularisations & $0.8 \%$ & $23.6 \%$ & $-22.8 \%$ & $0.8 \%$ & $11.8 \%$ & -11 \\
\hline Surgical & $0.8 \%$ & $0.8 \%$ & 0 & $0.8 \%$ & $0.3 \%$ & 0.5 \\
\hline Percutaneous & $0.0 \%$ & $22.9 \%$ & $-22.9 \%$ & 0.0 & $11.5 \%$ & -11.5 \\
\hline MACE-free survival & $94.2 \%$ & $71.2 \%$ & $23.0 \%$ & $94.2 \%$ & $83.1 \%$ & $11.1 \%$ \\
\hline $95 \% \mathrm{Cl}$ & 89.9 to 98.4 & 62.9 to 79.4 & 13.7 to 32.2 & 88.9 to 97.5 & 64.2 to 90.9 & 1.7 to 28.0 \\
\hline Angiography & $8.3 \%$ & $10.2 \%$ & $-1.9 \%$ & $10.3 \%$ & $14.1 \%$ & $-3.8 \%$ \\
\hline Total direct medical cost $(€)$ & 9969 & 9915 & 54 & 8065 & 7899 & 166 \\
\hline $95 \% \mathrm{Cl}$ & 8910 to 10504 & 8722 to 10449 & -1054 to 1296 & 7052 to 9463 & 6821 to 9591 & -1376 to 1487 \\
\hline
\end{tabular}




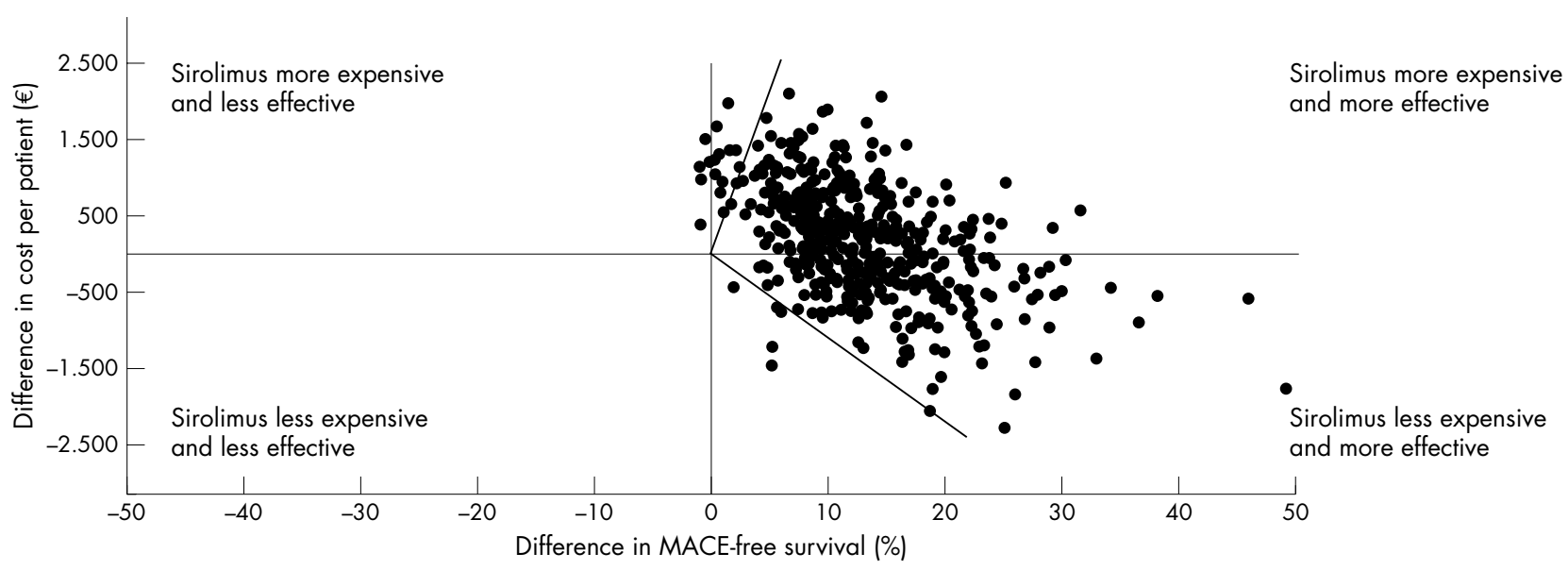

Figure 1 Estimates of cost and effects after a combination of bootstrapping and multivariate sensitivity analysis based on the normal distributions surrounding the estimates of the relative risks. MACE, major adverse cardiac events.

multivariate sensitivity analysis based on the normal distributions surrounding the estimates of the relative risks.

\section{DISCUSSION}

In this report, we analysed the balance between costs and effects in the RAVEL trial, while recognising the potential increase in interventions due to the oculostenotic reflex associated with protocol driven angiographic follow up. Two scenarios were presented: one with scheduled angiographic follow up, based on actual data from the RAVEL study, and one that corrected for the effect of protocol driven angiograms. The first scenario led to an estimated cost difference at the end of one year of $€ 54$ per patient and a cost per MACEfree survivor of $€ 234$. The second scenario led to an estimated cost difference of $€ 166$ at the end of one year and a cost per MACE-free survivor of $€ 1495$. Both point estimates seem to be well within a range of what may be considered acceptable from a societal standpoint.

These results need to be assessed in light of the study limitations. A first limitation is that the analysis was based primarily on data from the RAVEL study, which included only 238 patients with a primary end point of angiographic late loss at six months; MACE and resource utilisation were only secondary end points. Cost effectiveness was not a formal end point, mainly because of protocol mandated angiographic follow up, whose effect on outcomes was adjusted based on the BENESTENT II study. Our analysis of the costs and effects without angiographic follow up therefore has at least three sources of uncertainty. Firstly, there is the limited number of observations in the RAVEL study. Secondly, there is the estimate of effects of the oculostenotic reflex from the BENESTENT II study. Thirdly, there is the estimate of the costs that need to be subtracted as a result of this reflex. We considered all of these in our estimate leading to an upper $95 \%$ limit of $€ 61243$. These numbers might have been even higher considering that the patients in the BENESTENT II study differ from those in RAVEL. The difference in MACE-free survival, considering only the revascularisations that were labelled as being clinically driven, was very close to that of the combined analysis suggesting that this last potential source of error may have been relatively small.

An alternative approach would have been to recalculate costs and effects by excluding the events that were not clinically driven. We decided against this approach, since it would not account for the effects of the protocol driven angiograms on the rate of clinically driven angiograms and on the reintervention rate before month 5 and after month 7 .
The RAVEL study included a relatively non-complex group of patients, admitted for single lesion stenting (single vessel disease in $71 \%$ ) of short coronary stenosis (average lesion length $9.58 \mathrm{~mm}$ ). How the sirolimus eluting stent will perform in other patient populations with different risks of reintervention needs to be studied, in terms of not only efficacy but also cost effectiveness. Moreover, the RAVEL was an international study and no account was taken of differences in treatment patterns. Unit costs were estimated from one hospital and treated as if they were not surrounded by uncertainty. It was noted that the estimated differences in costs were highly dependent on the price difference between the sirolimus stents $(€ 2000)$ and the bare stents $(€ 672)$. As an example, when the price of the bare metal stent was set around $€ 500$, as it is in the UK, the total one year cost difference between the treatments was estimated at $€ 341$ in favour of bare stents, instead of $€ 166$.

A final important limitation concerns the outcome of the study. Sirolimus eluting stent implantation increased MACEfree survival, which is a combined end point of death, myocardial infarction, and repeat revascularisations. In practice it may reduce the need only for revascularisations. ${ }^{1-3}{ }^{10}$ Furthermore, it may be hypothesised that the antirestenotic effect of sirolimus stents may improve the rate of recurrent ischaemic symptoms and quality of life parameters. However, direct quality of life data have not been collected to determine the extent of the potential benefit of sirolimus stents in this regard. As such, cost per additional MACE-free survivor may not be the optimal way of expressing the balance between costs and effects in this scenario. In addition, the results obtained by calculating costs per MACE-free survivor, or costs per repeat procedure prevented, cannot be compared with the results of other health care interventions such as hip replacements. Given the lack of comparable outcomes, one may well ask the question how much society is prepared to pay to prevent repeat intervention during the first year after the initial intervention. However, when doing so, it may be important to recognise that the burden associated with the need for a repeat intervention may not be limited to a short period of anginal pain; one should also consider increased anxiety, disability and, most notably, an increased incidence of even more hospitalisations, some of which may result in repeat interventions.

\section{ACKNOWLEDGEMENTS}

The RAVEL trial was supported by a grant from Cordis, a Johnson \& Johnson company. 


\section{Authors' affiliations}

B A van Hout, Universitair Medisch Centrum, Julius Centrum, Utrecht, the Netherlands

P W Serruys, M J B M van den Brand, Erasmus Medical Centre,

Thoraxcentre, Rotterdam, the Netherlands

P A Lemos, Heart Institute (InCor)-University of Sao Paulo Medical School, Sao Paulo, Brazil

G-A van Es, W K Lindeboom, Cardialysis BV, Rotterdam, the Netherlands

M-C Morice, Institut Cardiovasculaire Paris Sud, Massy, France

\section{REFERENCES}

1 Morice MC, Serruys PW, Sousa JE, et al. A randomized comparison of a sirolimus-eluting stent with a standard stent for coronary revascularization. N Engl J Med 2002;346:1773-80.

2 Moses JW, Leon MB, Popma JJ, et al. Sirolimus-eluting stents versus standard stents in patients with stenosis in a native coronary artery. N Engl J Med 2003;349:1315-23.

3 Schofer J, Schluter M, Gershlick $A H$, et al. Sirolimus-eluting stents for treatment of patients with long atherosclerotic lesions in small coronary arteries: double-blind, randomised controlled trial (E-SIRIUS). Lancet 2003;362:1093-9

4 Serruys PW, van Hout B, Bonnier H, et al. Randomised comparison of implantation of heparin-coated stents with balloon angioplasty in selected patients with coronary artery disease (Benestent II). Lancet 1998;352:673-81.

5 Serruys PW, de Bruyne B, Carlier S, et al. Randomized comparison of primary stenting and provisional balloon angioplasty guided by flow velocity measurement. Doppler endpoints balloon angioplasty trial Europe (DEBATE) II study group. Circulation 2000;102:2930-7.

6 Serruys PW, Unger F, Sousa JE, et al. Comparison of coronary-artery bypass surgery and stenting for the treatment of multivessel disease. N Engl J Med 2001;344:1117-24.

7 Van den Brand $M$, van Halem C, van den Brink F, et al. Comparison of costs of percutaneous transluminal coronary angioplasty and coronary bypass surgery for patients with angina pectoris. Eur Heart J 1990;1 1:765-71.

8 Ruygrok PN, Melkert R, Morel MA, et al. Does angiography six months after coronary intervention influence management and outcome? Benestent II investigators. J Am Coll Cardiol 1999;34:1507-11.

9 Efron B, Tibshirani RJ. An introduction to the bootstrap. Monographs on statistics and applied probability, vol 57. London: Chapman and Hall, 1993.

10 Lemos PA, Serruys PW, Sousa JE. Drug-eluting stents: cost versus clinical benefit. Circulation 2003;107:3003-7.

\section{IMAGES IN CARDIOLOGY}

\section{Intracardiac extension of a large cell undifferentiated carcinoma of lung}

doi: $10.1136 /$ hrt.2004.039354

A 60 year old male presented with complaints of cough, dyspnoea, reduced appetite, and a history of 70 pound $(30 \mathrm{~kg})$ weight loss over the previous year.

Chest radiograph showed complete opacification of the left hemithorax with only minimal aeration of the most cephaloid lung apex.

A computed tomographic (CT) scan (below left) of the chest showed a $10 \mathrm{~cm}$ mass in the posterior left lower lobe with invasion into the left atrium by way of the left inferior pulmonary vein. There was left basilar lung atelactasis with a small left pleural effusion. The panel shows the lung mass and tumour extension into the left atrium $(\mathrm{AL}$, atelactatic lung; LA, left atrium; LV, left ventricle; RA, right atrium; RV, right ventricle, $\mathrm{T}$, tumour).

A bronchoscopy was performed. This showed complete occlusion of the left main stem bronchus secondary to an endobronchial lesion arising from the lower left bronchial tree.

Bronchoscopic biopsy was performed. This showed poorly differentiated carcinoma with large, hyperchromatic,

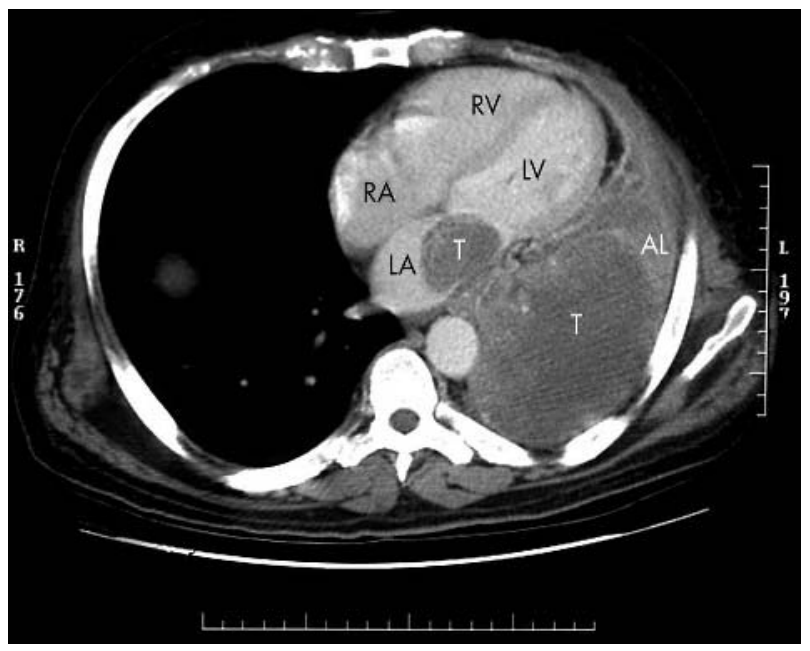

pleomorphic nuclei with prominent nucleoli and coarse chromatin (below right: haematoxylin and eosin, $\times 40$ magnification). The cells had pale, vacuolated cytoplasm with indistinct cell borders. Features to suggest squamous cell carcinoma, such as sharp cytoplasmic borders and cytoplasmic keratinisation, were not seen. Features diagnostic of adenocarcinoma, such as intracytoplasmic mucin, glandular differentiation, and a papillary growth pattern, were not identified. These findings were consistent with a large cell undifferentiated carcinoma of lung.

The patient was treated with carboplatin and paclitaxel chemotherapy.

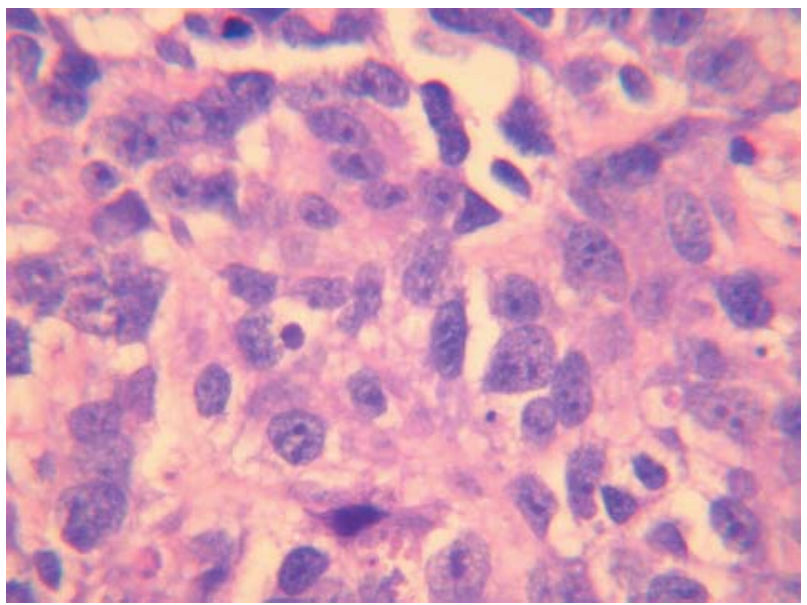

